\title{
Spectral-Domain Optical Coherence Tomography
} Features of Acute Syphilitic Posterior Placoid

\section{Chorioretinitis: The Role of Autoimmune Response in Pathogenesis}

\author{
Pedro Brito Susana Penas Ângela Carneiro \\ Jorge Palmares F. Falcão Reis
}

Department of Ophthalmology, Hospital S. João, University of Porto, Porto, Portugal

\section{Key Words}

Syphilis · Placoid - Chorioretinitis - Spectral-domain optical coherence tomography Beta2 glycoprotein

\begin{abstract}
Purpose: Syphilis is an infectious disease that can cause a wide variety of ocular signs. One of the rarest manifestations of ocular syphilis is acute syphilitic posterior placoid chorioretinitis (ASPPC). We report on the spectral-domain optical coherence tomography (SD-OCT) features of a case diagnosed with unilateral ASPPC.
\end{abstract}

Methods: A 64-year-old man presented with a sudden loss of visual acuity (VA) in the right eye. His only clinical sign was a large, geographic, yellow-white lesion centered on the right fovea. Our patient was studied with SD-OCT on presentation and during followup, as well as with fluorescein and indocyanine green angiography, electrophysiological study, and serologic and autoimmune screening.

Results: Laboratory workup revealed positive serology for active syphilis and elevated anti-beta2 glycoprotein I antibodies. SD-OCT showed a marked distortion of both the choroidal and outer retinal architecture. After treatment, best-corrected VA improved to 20/25. Pattern electroretinography displayed a severe reduction of P50 amplitude, which improved in late follow-up. Six months after presentation, VA was 20/25 and anti-beta2 glycoprotein I antibodies returned to normal levels.

Conclusions: Our findings are compatible with immunologically mediated temporary physiological impairment of the neuroretina, since the changes seen by SD-OCT could not have normalized if they were due to anatomical injury. The results of our study 


\begin{tabular}{|c|c|c|c|}
\hline ase Reports in & $\begin{array}{l}\text { Case Rep Ophthalmol 2011;2:39-44 } \\
\text { DOI: } 10.1159 / 000324086\end{array}$ & $\begin{array}{l}\text { Published online: } \\
\text { January } 25,2011\end{array}$ & \begin{tabular}{|l} 
@ 2011 S. Karger AG, Basel \\
ISSN $1663-2699$ \\
www.karger.com/cop
\end{tabular} \\
\hline
\end{tabular}

provide clues to understanding the pathogenesis of this disease and allow us to define a characteristic temporal sequence of events in ASPPC.

\section{Introduction}

Syphilis is a chronic infectious disease caused by the spirochete Treponema pallidum, which can affect all structures of the eye. Some manifestations include interstitial keratitis, iritis, vitritis, chorioretinitis, serous retinal detachment and papillitis [1]. Due to the wide spectrum of possible signs, syphilis is often named as the great masquerader of ocular diseases [2]. Acute syphilitic posterior placoid chorioretinitis (ASPPC) is a rare manifestation of syphilis, first described by Gass et al. in 1990 [3]. Even though some theories have been proposed, the pathogenesis of this entity still remains unknown $[4,5]$. In this case report, we describe the spectral-domain optical coherence tomography (SDOCT) findings along with the clinical evolution of a patient diagnosed with ASPPC.

\section{Case Report}

A 64-year-old male presented with a 1-day history of sudden visual loss in the right eye (RE). There were no other eye complaints. When asked about systemic complaints, he referred general weakness, but denied other symptoms. He was not taking any medications and his medical history was irrelevant. The patient was a smoker but denied illicit drug abuse. His best-corrected visual acuity (BCVA) was hand movement in the RE and 20/25 on the left eye (LE). Slit lamp examination of the anterior segment was unremarkable. Fundoscopic examination revealed a yellow-white, subretinal, placoid lesion in the macula of the RE (fig. 1), but there was no vitritis, papillitis, retinal hemorrhages or cotton wool spots. Fluorescein angiography revealed a lesion of 4-5 disk diameters in size, with well-defined borders and late-staining hyperfluorescence (fig. 1). SD-OCT (Spectralis; Heidelberg Engineering, Germany) was performed based on the technique first described by Spaide et al. [6]. We used a $30^{\circ}$ horizontal line passing directly through the center of the fovea. The obtained image was averaged from 100 scans and revealed diffuse choroidal infiltration with reduction of the diameter and number of the usual vascular spaces when compared to the LE, as well as an ill-defined photoreceptor inner/outer segment junction and absent external limiting membrane (ig. 2).

Since we did not have a clear diagnosis, no treatment was initiated. The patient returned for a scheduled indocyanine green angiography which, for technical reasons, was possible only 3 weeks later. Examination of the anterior segment remained normal in both eyes and the macular lesion had disappeared, but VA was still hand movements in the RE. Laboratory workup included cerebral CT scan, electrocardiogram, full blood count with erythrocyte sedimentation rate (ESR), liver enzymes, electrolytes, autoimmunity screening (antinuclear antibody, anticardiolipin antibodies and rheumatoid factor) and serologic screening for toxoplasmosis, syphilis, cytomegalovirus and herpes virus. Significant findings included elevated ESR ( 3 times the normal value) and IgM anti-beta2 glycoprotein I antibody (B2GPI), which was also 3 times the normal value. Serologic tests were positive for syphilis, both VDRL (1/16 dilutions) and Treponema pallidum particle agglutination assay, indicating active infection. Electrophysiological study was performed by visual evoked potentials which revealed a moderate P100 amplitude reduction, and pattern electroretinography (ERG) showed a marked reduction of $\mathrm{P} 50$ amplitude, compatible with macular dysfunction. These results allowed us to make the diagnosis of ASPPC. Unfortunately, the patient missed the next visit and returned only 1 month later for rescheduled observation. The BCVA had improved to 20/40 in the RE and remained 20/25 in the LE. After checking the patient's medical records, we found that in the last 2 weeks he had been taking oral dexamethasone and amoxicillin due to a throat inflammation. Follow-up SD-OCT showed a slight improvement of the choroidal pattern as well as a completely restored stratification of the outer retinal layers (fig. 3 ). Treatment was initiated with intramuscular benzopenicillin 2.4 million units once a week during 3 weeks, and we referred the patient to an infectiologist for systemic examination. Lumbar puncture was negative for syphilis. HIV, hepatitis B and C tests were negative. The patient returned 1 
week later with a BCVA of 20/25 in the RE, anterior segment examination remained normal as well as fundoscopy. Repeated fluorescein angiography was normal. He returned 1 month later and his BCVA had decreased to 20/40, but ophthalmologic examination revealed no other changes. Follow-up serologic testing revealed a decrease of VDRL to $1 / 8$ dilutions, and anti-B2GPI antibodies were only slightly reduced compared with the previous level. SD-OCT, pattern ERG and multifocal ERG were scheduled. Additional treatment was initiated with amoxicillin 1.5/day and oral dexamethasone 1 $\mathrm{mg} / \mathrm{kg} /$ day. Two weeks later, BCVA in the RE had improved to 20/25, and pattern ERG revealed a slight improvement in P50 amplitude, but multifocal ERG indicated persistently diminished wave amplitude in all stimulated zones. SD-OCT imaging was similar to the previous examination. Antibiotic therapy was stopped and corticosteroid was tapered over the course of 1 month. In the following 3 months, there were no other relapses, a repeated autoimmunity screening revealed normal levels of IgM antiB2GPI antibodies.

\section{Discussion}

The term ASPPC defines a variant of syphilitic chorioretinitis characterized by a large, solitary, placoid macular lesion that was thought to occur primarily in immunocompromised individuals. The first reported cases were indeed positive for HIV, and later cases were described in patients immediately after corticosteroid treatment which seems to suggest an immune modulation of the disease $[3,5,7]$. Recently, a few cases were reported in immunocompetent individuals [8-10]. Our patient was also immunocompetent, and it is now well-known that ASPPC can occur independently of the immune status of the patient, but the pathogenesis of this entity still remains unknown. In our patient, the angiographic and SD-OCT findings clearly point to a pathogenic process at the level of the choroid-retinal pigment epithelium complex which had repercussions in the photoreceptor internal/external segment junction layer and the external limiting membrane (fig. 2). Previous reports, using time-domain OCT imaging, identified increased retinal thickness and subretinal fluid [9-11]. Our findings were different and to our knowledge, this is the first report on the SD-OCT features of ASPPC. The retinal changes documented by SD-OCT imaging could not have been due to anatomical injury, as we verified full retinal stratification normalization even before adequate treatment had been completed and VA had not yet fully recovered. These findings point to a temporary physiological impairment of the neuroretina.

Another interesting result in our patient is the increased level of IgM anti-B2GPI. These antibodies occur in autoimmune (lupus, primary antiphospholipid syndrome) as well as in infectious diseases (leptospirosis, syphilis) [12]. B2GPI is an apolipoprotein that binds to cardiolipin, which is found in the inner mitochondrial membrane. Some functions regulated by cardiolipin include anticoagulation and mitochondrial metabolism [13]. The presence of the treponemes in the choroid probably leads to the formation of anti-B2GPI antibodies which, in turn, could cause temporary focal choroidal thrombosis and altered retinal pigment epithelium metabolism, resulting in impaired photoreceptor function. In the available ophthalmologic literature, we could not find a description of the normal choroidal SD-OCT pattern in healthy individuals. This may be due to the fact that before Spectralis OCT was available, there was no OCT device that allowed reliable and detailed choroidal imaging, or it could also be that normal choroid pattern is variable between individuals. Even if this is the case, a certain degree of symmetry in choroid anatomy is to be expected between the eyes of the same individual. The incomplete recovery of the choroidal vasculature, seen in the SD-OCT images obtained after treatment was completed, probably reflects deposited fibrin, platelets and inflammatory cellular debris, and a certain amount of permanent ischemic damage is expected. This 
theory could explain the fundoscopic, OCT and electrophysiologic signs of ASPPC. Therefore, anticardiolipin antibodies should be a part of the laboratory workup for ASPPC. This case also confirms a sequential pattern which was already perceptible in previous reports [5]. First there is an anatomical recovery with the fundoscopic lesions disappearing in the first 1-2 weeks independently of syphilis treatment, and complete visual recovery usually occurs after about 1 month and requires adequate treatment $[3,5$, $9,11]$; this stage is followed by a delayed and incomplete electrophysiogical recovery [9].

Finally, it is interesting to note that our patient had a loss of VA a few weeks after penicillin treatment. He had been taking corticosteroids for an unrelated problem during 2 weeks and stopped abruptly. This led us to believe that the visual loss was probably due to a relapse of the inflammatory activity. It seems that the most appropriate treatment for ASPPC requires both antibiotic treatment (parenteral penicillin regimen determined by severity of syphilitic disease, i.e. neurosyphilis, should always be investigated) and corticosteroid treatment tapered over a period of at least 1 month. In this way, we are fighting against both the infectious agent (Treponema pallidum) and the immune reaction it causes (anticardiolipin antibodies, inflammatory response).

ASPPC can cause the first symptoms that bring the patient to the hospital, and this gives the ophthalmologist an important role in the detection and treatment of this disease. Adequate treatment results in good visual recovery.

\section{Disclosure Statement}

No sponsorship or funding arrangements relating to research and no conflicts of interest to declare. 


\begin{tabular}{l|l|l|l} 
Case Reports in & $\begin{array}{l}\text { Case Rep Ophthalmol 2011;2:39-44 } \\
\text { DOI: } 10.1159 / 000324086\end{array}$ & $\begin{array}{l}\text { Published online: } \\
\text { January 25, 2011 }\end{array}$ & $\begin{array}{l}\text { ○ 2011 S. Karger AG, Basel } \\
\text { ISSN 1663-2699 } \\
\text { www.karger.com/cop }\end{array}$ \\
\hline
\end{tabular}

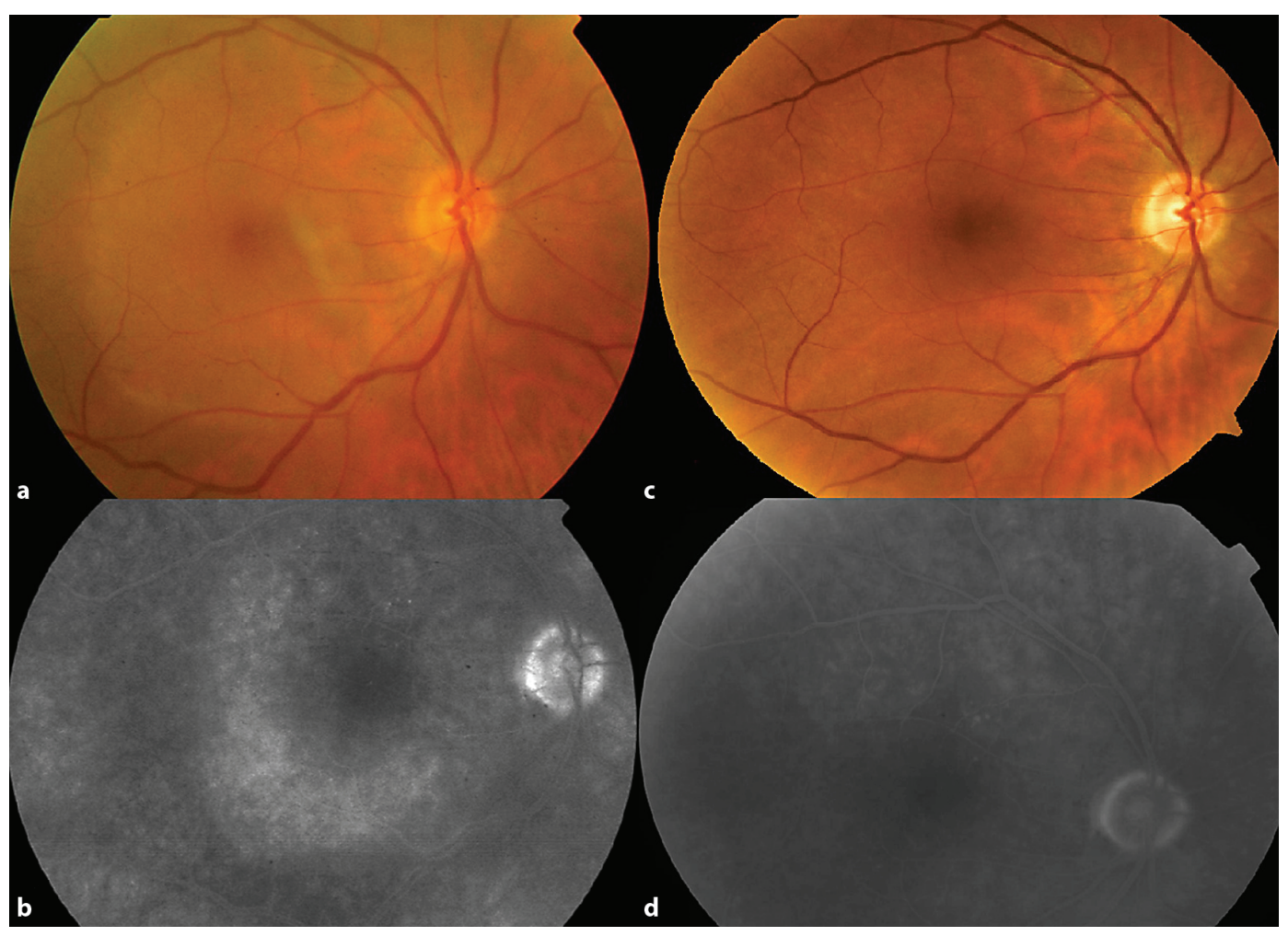

Fig. 1. Fundus image of the RE showing a typical placoid macular lesion of ASPPC (a). Late-staining hyperfluorescence seen on angiography (b). Normal fundus of the RE, 2 weeks after presentation (c). Normal angiography after completion of treatment $(\mathbf{d})$.
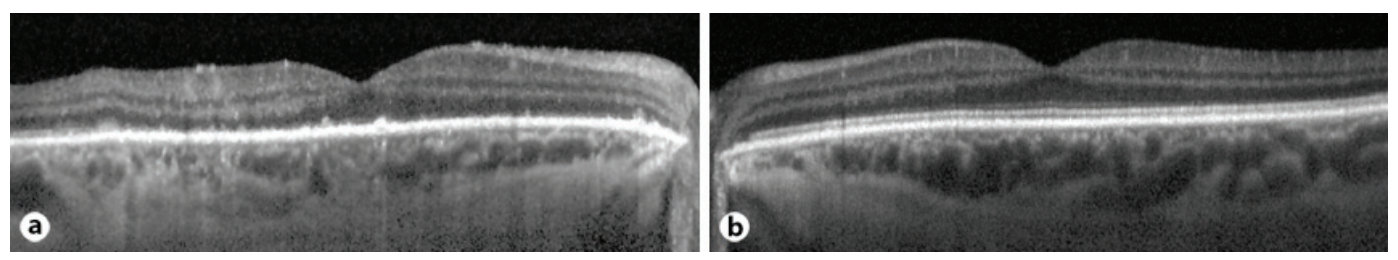

Fig. 2. SD-OCT of the right macula (a) showing absence of the inner/outer photoreceptor segment junction layer as well as an absent external limiting membrane. Choroidal detail reveals irregular, diffuse infiltration of the choroidal vasculature (b). SD-OCT of the left macula showing normal retinal stratification and well-defined, numerous choroidal vascular spaces. 


\begin{tabular}{l|l|l|l} 
Case Reports in & $\begin{array}{l}\text { Case Rep Ophthalmol 2011;2:39-44 } \\
\text { DOI: } 10.1159 / 000324086\end{array}$ & $\begin{array}{l}\text { Published online: } \\
\text { January 25, 2011 }\end{array}$ & $\begin{array}{l}\text { ○ 2011 S. Karger AG, Basel } \\
\text { ISSN 1663-2699 } \\
\text { www.karger.com/cop }\end{array}$ \\
\hline
\end{tabular}
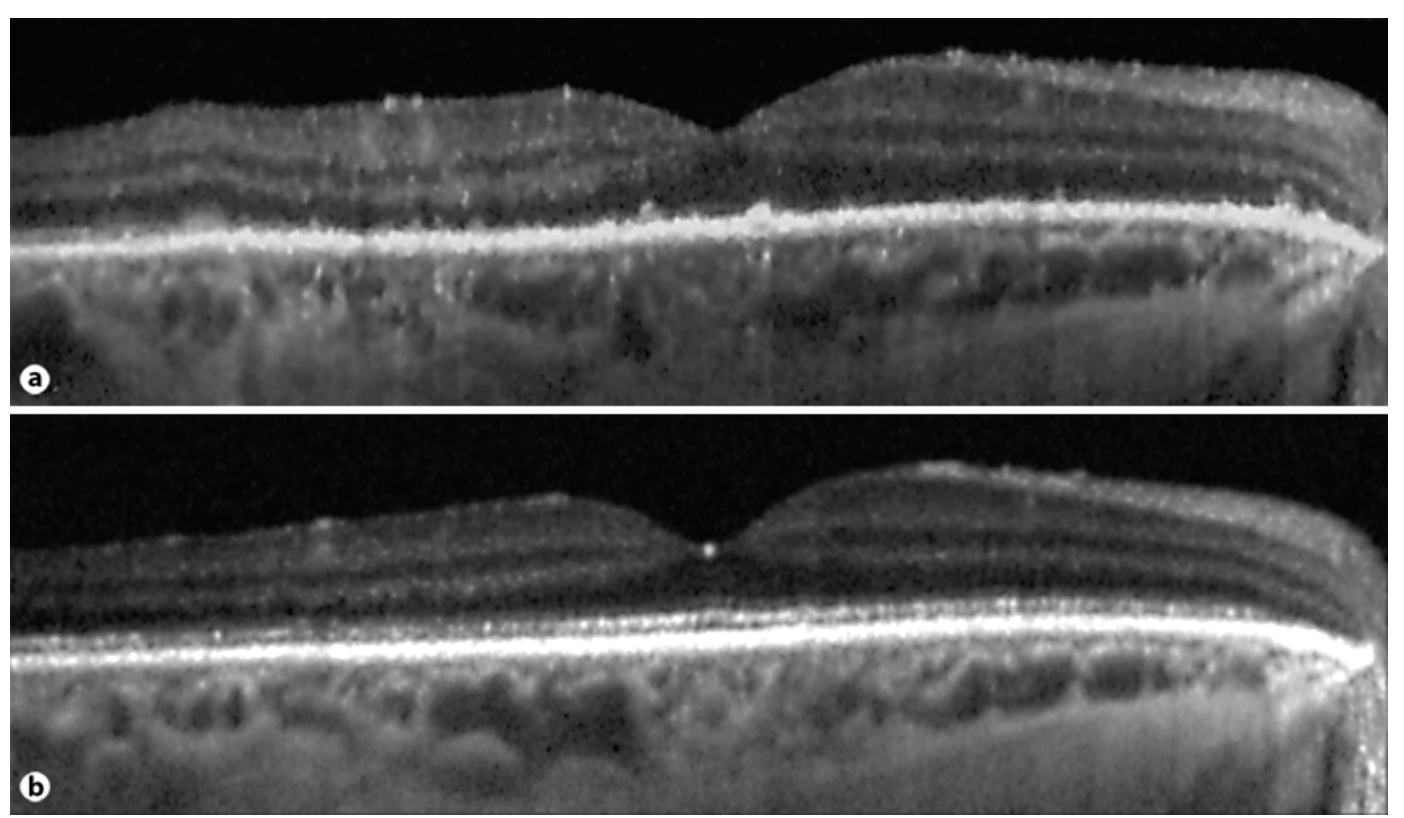

Fig. 3. SD-OCT image comparison before (a) and after treatment completion (b). The inner/outer segment junction is now visible in the full extent of the scan. Choroidal detail reveals slightly improved vascular definition, better noticed in the area temporal to the fovea.

\section{References}

1 Kiss S, Damico FM, Young LH: Ocular manifestations and treatment of syphilis. Semin Ophthalmol 2005;20:161-167.

-2 Aldave AJ, King JA, Cunningham ET Jr: Ocular syphilis. Curr Opin Ophthalmol 2001;12:433-441.

-3 Gass JD, Braunstein RA, Chenoweth RG: Acute syphilitic posterior placoid chorioretinitis. Ophthalmology 1990;97:1288-1297.

4 de Souza EC, et al: Unusual central chorioretinitis as the first manifestation of early secondary syphilis. Am J Ophthalmol 1988;105:271-276.

5 Zamani M, Garfinkel RA: Corticosteroid-induced modulation of acute syphilitic posterior placoid chorioretinitis. Am J Ophthalmol 2003;135:891-894.

-6 Spaide RF, Koizumi H, Pozzoni MC: Enhanced depth imaging spectral-domain optical coherence tomography. Am J Ophthalmol 2008;146:496-500.

7 Erol N, Topbas S: Acute syphilitic posterior placoid chorioretinitis after an intravitreal triamcinolone acetonide injection. Acta Ophthalmol Scand 2006;84:435.

8 Yoo C, et al: Atypical acute syphilitic posterior placoid chorioretinitis. Korean J Ophthalmol 2009;23:108-111.

$\$ 9$ Joseph A, et al: Syphilitic acute posterior placoid chorioretinitis in nonimmuno-compromised patients. Eye (Lond) 2007;21:1114-1119.

10 Chen J, Lee L: Posterior placoid chorioretinitis: An unusual ocular manifestation of syphilis. Clin Ophthalmol 2008;2:669-673.

-11 Meira-Freitas D, et al: Optical coherence tomography and indocyanine green angiography findings in acute syphilitic posterior placoid choroidopathy: case report. Arq Bras Oftalmol 2009;72:832-835.

12 Santiago M, et al: Anti-beta2 glycoprotein I and anticardiolipin antibodies in leptospirosis, syphilis and Kalaazar. Clin Exp Rheumatol 2001;19:425-430.

13 Schlame M, Rua D, Greenberg ML: The biosynthesis and functional role of cardiolipin. Prog Lipid Res 2000;39:257-288. 